\title{
Diagnostic Studies of Infectious Bronchitis Disease in Broilers using Pathological and Molecular Investigations in Kaliobeya Governorate, Egypt.
}

\author{
Heba M Abdel- Ghany ${ }^{1}$ and Nora M Elseddawy ${ }^{2}$ \\ 1 Pathology Department, Faculty of Veterinary Medicine, Zagazig University, Egypt. \\ ${ }^{2}$ Pathology Department, Faculty of Veterinary Medicine, Zagazig University, Egypt.
}

Correspondence Author: Heba M Abdel- Ghany, Pathology Department, Faculty of Veterinary Medicine, Zagazig University, , Egypt. E-mail: Heba.vet@yahoo.com

Received date: 12 November 2018, Accepted date: 10 January 2019, Online date: 25 January 2019

Copyright: (c) 2019 Heba M Abdel- Ghany et al., This is an open-access article distributed under the terms of the Creative Commons Attribution License, which permits unrestricted use, distribution, and reproduction in any medium, provided the original author and source are credited.

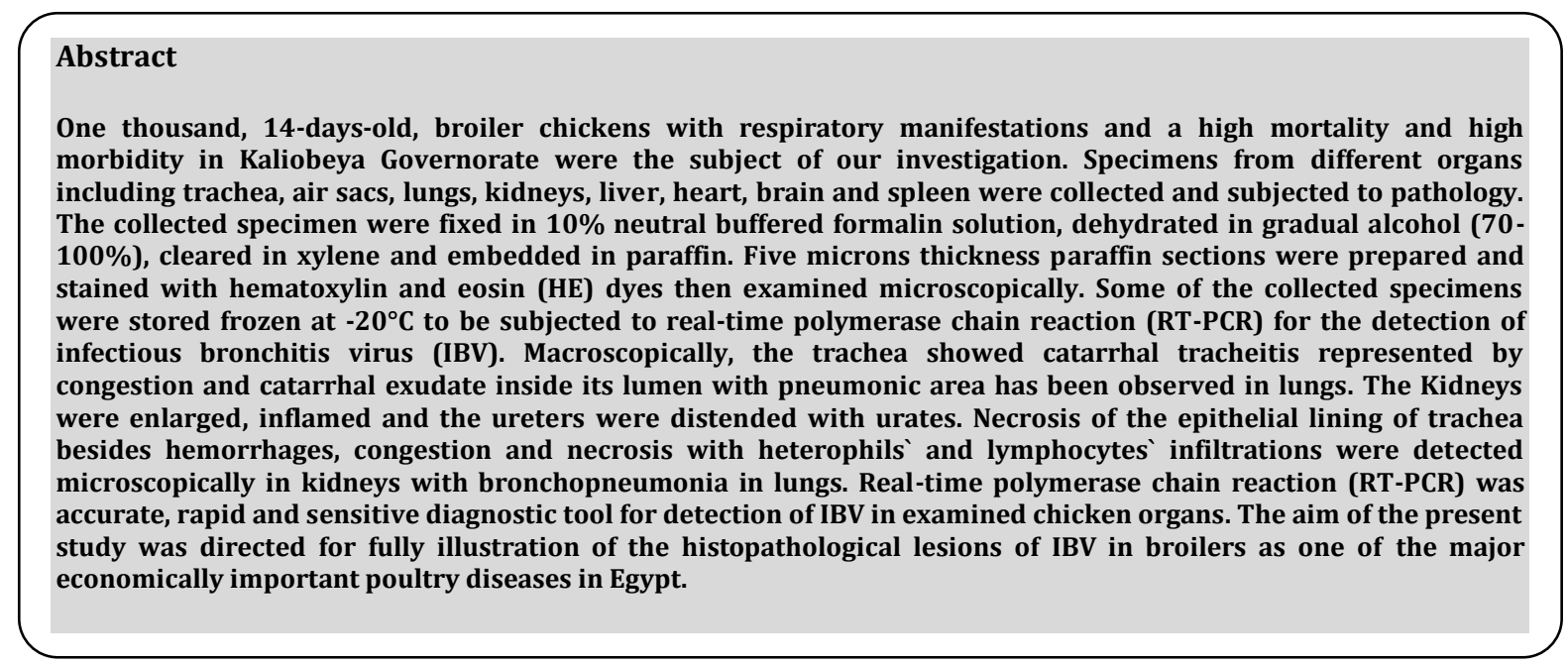

Key words: $I B V$, broilers, Coronaviridae, pathology, $P C R$

\section{INTRODUCTION}

Infectious bronchitis (IB) is an acute, highly contagious viral disease of respiratory and urogenital system of chicken affecting all ages of both broilers and layers. It has a great economic loss to the poultry industry worldwide causing high mortality, poor body weight gain in broilers and decreased egg production in layers. The virus is transmitted by shedding of the viral particle in ocular and nasal discharge, as well as faecal droplets from infected poultry. Contaminated objects and utensils may play role in transmission of the virus from a flock to another [1]. Infectious bronchitis disease is caused by infectious bronchitis virus (IBV), an enveloped RNA virus. It has been classified under the Gamma Coronaviruses Genus within the Coronaviridae Family [2]. The IBV targets primarily the epithelial cells of the respiratory tract of the chickens [3]. The disease was reported firstly in 1931 in the USA as a respiratory disease [4]. Some IBV strains are nephropathogenic infecting kidneys and reproductive tis sues [5].Chickens of all ages are susceptible to IBV infection; the severity of the disease is noticed in young chicks, compared to adults and resistance to infection increases with increasing the age. The morbidity rate can reach $100 \%$ but the mortality rate depends on the presence of secondary infections, immune status, flock age, environmental factors and management. In young chickens, the mortality rate is typically $25-30 \%$ but it can approach $80 \%$ depending on the virulence of the strain [1].Some strains of IBV cause acute nephritis and urolithiasis associated with the high mortality of infected chickens, more ever, the disease is a predisposing factor for secondary bacterial infection resulting in high mortality and morbidity rate [6]. Replication of IBV occurs primary in tracheal mucosa [7], after replication in tracheal mucosa, viraemia and secondary replication are found in other respiratory tissues as nose, lungs and air sacs and in non-respiratory tissues such as kidneys, gastrointestinal tract, oviduct, and testes [8]. Clinically, 
most birds infected with IBV showed depression, dullness, reduced feed consumption, ruffled feather, increase water consumption, huddle together under heat source, coughing, gasping, sneezing, tracheal rales, occulonasal discharge and mild swollen of sinus [9, 10]. The pathological changes observed grossly include: congestion, edema with serous catarrhal exudate in tracheal lumen. Kidneys were enlarged, pale, inflamed, mottled with distended tubules with urates. Congestion with focal area of consolidation has been observed in lungs. Air sacs may be foamy during the acute infection and then may become cloudy and contain yellow caseous exudates $[6,11]$. The microscopic examination of trachea was loss of cilia and sloughing of epithelial cells, serous exudation inside the lumen with hypertrophy of mucosal gland with heterophils` and lymphocytes` infiltrations in the mucosa. Kidneys showed interstitial lymphocytic and heterophilic infiltrations, tubular degeneration, necrosis; edema of Bowman's capsule, dilated renal tubules with urate crystals and cast, congestion and hemorrhage. Lungs revealed congestion, hemorrhage, fibrin exudate and infiltration of lymphocytes and heterophils. The air sac revealed edema, epithelial desquamation and fibrinous exudate. The liver showed congestion, degeneration and necrosis of hepatic cells with distortion of hepatic cords and infiltration of inflammatory cells [12].Vaccination is commonly adopted but outbreaks continue to occur worldwide due to new variants strains continue to emerge [13]. There is little or no cross-protection between different serotypes of IBVs leading to immune failures and making the disease difficult to control [14]

\section{MATERIALS AND METHODS}

\section{Collection and preparation of tissue specimens}

One thousand, 14-days-old broiler chickens with respiratory disease and high mortality and high morbidity $80 \%$ in Kaliobeya Governorate, Egypt were the subject of our investigation. Specimens from different organs exhibiting gross lesions were collected and subjected to histopathological examination and RT-PCR.

\section{Pathological examination}

The collected specimens including trachea, air sacs, lungs, kidneys, liver, heart, brain and spleen were fixed in $10 \%$ neutral buffered formalin solution, dehydrated in gradual alcohol (70-100\%), cleared in xylene and embedded in paraffin. Five microns thickness paraffin sections were prepared and stained with hematoxylin and eosin (HE) dyes then examined microscopically [15]. Molecular detection of IBV by PCR

DNA extraction was performed according to QIAamp Viral RNA Mini extraction kits (Qiagen, Germany). Oligonucleotide primer / probe sequences (Metabion, Germany), AIBV-fr 5'-ATGCTCAACCTTGTCCCTAGCA-3', AIBV-as 5'-TCAAACTGCGGATCA-TCACGT-3 and AIBV-TM 5'-FAM-TTGGAAGTAGAGTGACGCCCAAACTTCA-TAMRA-3' were selected from a previously published paper [16]. Real-time PCR was performed with a final volume of $25 \mu \mathrm{L}$ of the following reaction mixture: $12.5 \mu \mathrm{L}$ QuantiTect Probe RT-PCR Master Mix (2X) (Sigma, UK), $0.5 \mu \mathrm{L}$ of each primer (50 pmole), $0.25 \mu \mathrm{L}$ QuantiTect RT Mix, $7 \mu \mathrm{L}$ template DNA and 4.25.5 $\mu \mathrm{L}$ RNase Free Water. .Real time PCR thermal cycling was applied according to a previously published paper [16] with some modification; reaction mixtures were incubated at $50^{\circ} \mathrm{C}$ for 30 min for reverse transcription followed by one cycle of primary denaturation at $95^{\circ} \mathrm{C}$ for 15 min then 40 cycles of secondary denaturation at $95^{\circ} \mathrm{C}$ for $15 \mathrm{~s}$ and annealing and final extension at $60^{\circ} \mathrm{C}$ for $45 \mathrm{~s}$.

\section{RESULTS}

\section{Clinical signs}

The most observed clinical signs were depression, reduced feed consumption, ruffled feather, huddle together under heat source, coughing, gasping, sneezing and tracheal rales.

\footnotetext{
Pathological findings

Macroscopically, the pathological changes observed grossly included: congestion and catarrhal exudate in tracheal lumen. The airsac was cloudy with fibrin thread. Pneumonic area has been observed in lung. The kidneys were enlarged and inflamed (Fig 1 A). Microscopically, the trachea showed catarrhal tracheitis represented by congestion of blood vessels in mucosa and submucosa with infiltration of heterophils and loss of cilia (Fig 1 B). Necrosis of epithelial lining replaced by hemorrhage and heterophils infiltration with edema in submucosa were also detected (Fig $1 \mathrm{C}$ ). The air sac showed thickening in its wall with fibrin threads and lymphocytes`and heterophils`infiltrations (Fig 1 D). The lung showing thickening of interalvealar septa with hemorrhage, fibrin exudate and heterophils' infiltrations (Fig 2E). The kidneys showed congestion of peritubular blood vessels with hemorrhage (Fig 2 F). Dilation of Bowman's capsule with necrosis of some renal tubules characterized by pyknosis, karyorhexsis and karyolysis were detected (Fig 2G). Tubular degeneration represented by cloudy swelling and vacuolar degeneration (Fig $2 \mathrm{H}$ ). Interstitial lymphocytic and fibroblast infiltrations were observed (Fig 3 I). Cellular and hyaline cast inside the lumen of some renal tubules were observed (Fig $3 \mathrm{~J}$ ). The liver showed congestion of central vein, hepatic sinusoides and portal vein with thickining and hyalinization in its wall beside focal aggrgation of lymphocytes and heterophils (Fig 3k). Necrosis of some hepatic cells replaced by lymphocytes`and heterophils`infiltartions with distotion of hepatic cords were recorded (Fig 3 L). The heart showed thickening in the pericardium with existence of lymphocytes infiltration and fibrin threads. Congestion of blood vessels with intermuscular edema between muscle fibers were detected (Fig $4 \mathrm{M}$ ). Focal aggregations of lymphocytes`and heterophils` infiltrations with hemorrhage were observed (Fig 4 N).. The brain showed perivascular lymphocytic cuffing with degenerted neurons. Satellitosis and neurophagia were detected (Fig 4O). Spleen showed thickening and hyalinization of central arterioles with endotheliosis and depletion of lymphocytes (Fig 4P)
} 


\section{RT-PCR results}

Indeed, real time RT-PCR offer a sensitive, rapid, and accurate results thus enabling the detection of IBV in examined organs of infected broilers within a short time (data not shown).
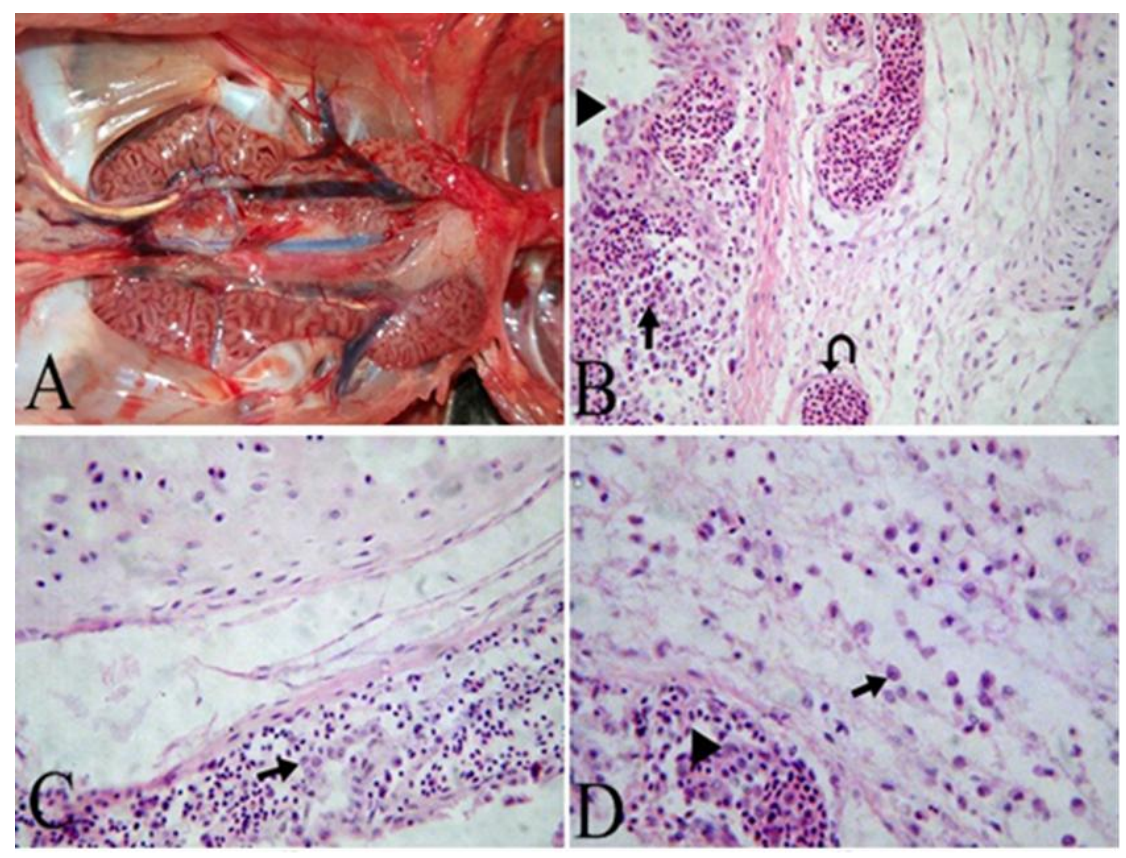

Fig. 1: (A) Kidneys were enlarged and inflamed B: The trachea showing catarrhal tracheitis represented by congestion of blood vessels in submucosa (oblique arrow) with infiltration of heterophils (arrow) and loss of cili a)arrow head) HE x1200. C: The trachea showing necrosis of epithelial lining replaced by hemorrhage and heterophils infiltration (arrow) HE x1200. D: The air sac showing thickening in its wall with fibrin threads and lymphocytes`and heterophils` infiltrations (arrow). Congestion of blood vessels (aroow head) HE x1200.

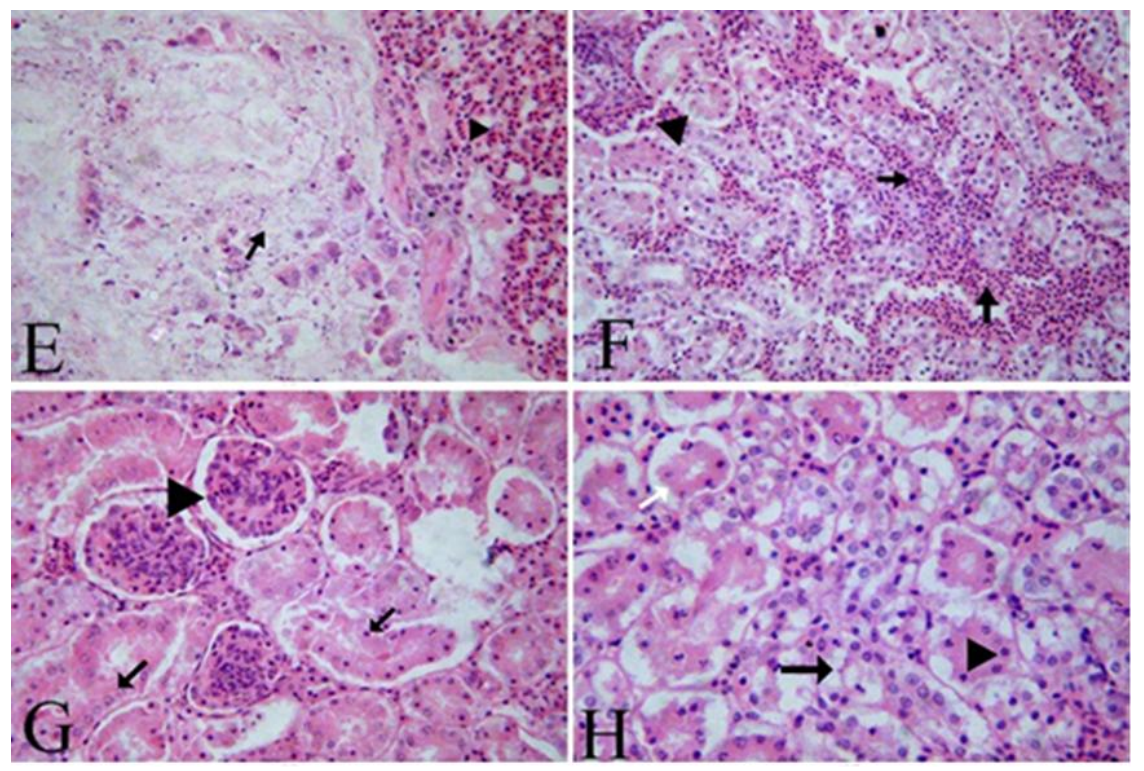

Fig. 2: (E) The lung showing thickening of interalvealar septa with hemorrhage (arrow head), fibrin exudate with heterophils` infiltrations ' in lung tissue (arrow) HE x1200. (F) The kidneys showing congestion of peritubular blood vessels (arrow head) with hemorrhage (arrow) HE x1200. (G) The kidney showing dilation of Bowman's capsule (arrow head) with necrosis of some renal tubules characterized by pyknosis and karyolysis (arrow) HE x1200. (H) The kidney showing vacuolar degeneration (arrow) and necrosis of the epithelial lining of some renal tubules (arrow head) with presence of cellular cast inside the lumen of renal tubules (white arrow) HE x1200. 


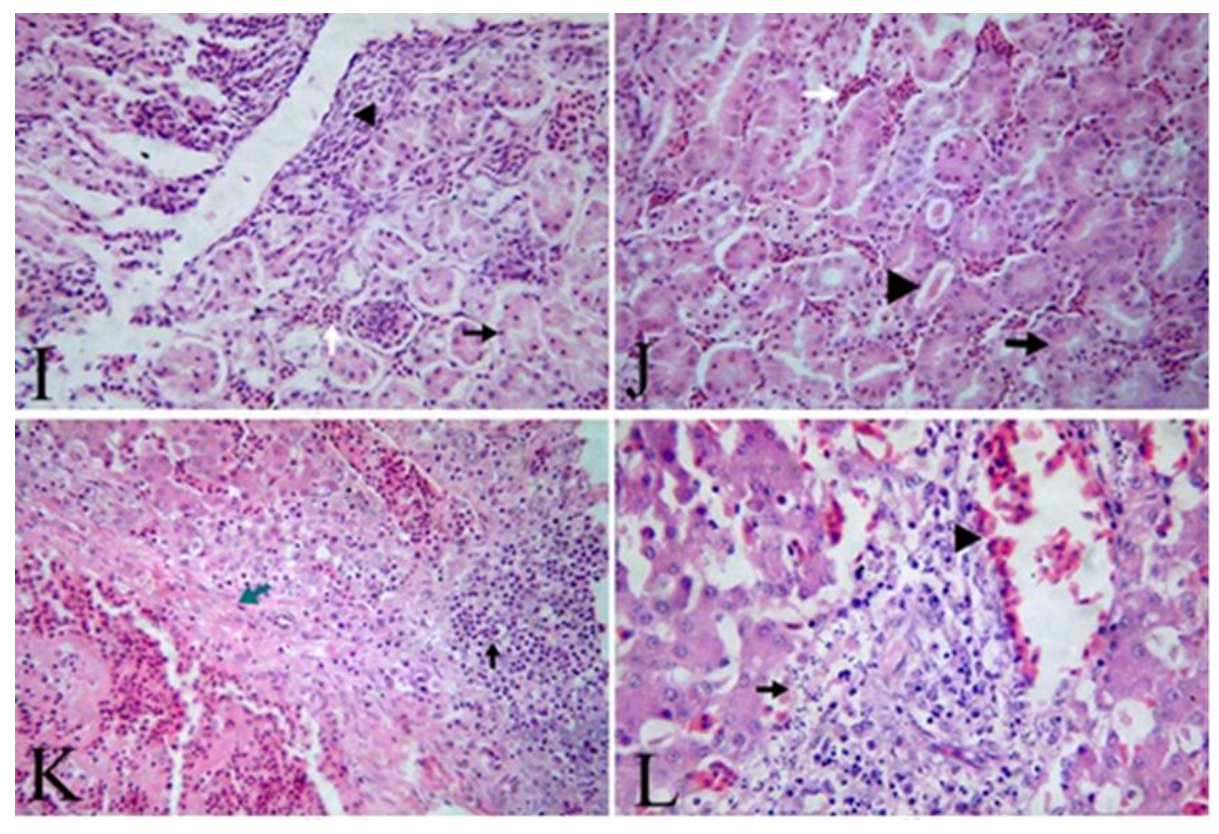

Fig. 3: (I) The kidney showing interstitial lymphocytic and fibroblast infiltrations (arrow head) with congestion of peritubular blood vessels (white arrow) HE x1200. (J) The kidney showing hyaline cast inside the lumen of some renal tubules (arrow head) with cloudy swelling (arrow) HE x1200.( K) The liver showing congestion of the portal vein with thickining and hyalinization in its wall (green arrow) beside focal aggrgation of lymphocytes and heterophils (black arrow) HE x1200. (L) The liver showing necrosis of some hepatic cells replaced by lymphocytes`and heterophils`infiltartions (arrow) with congestion of central vein (arrow head) HE x1200.

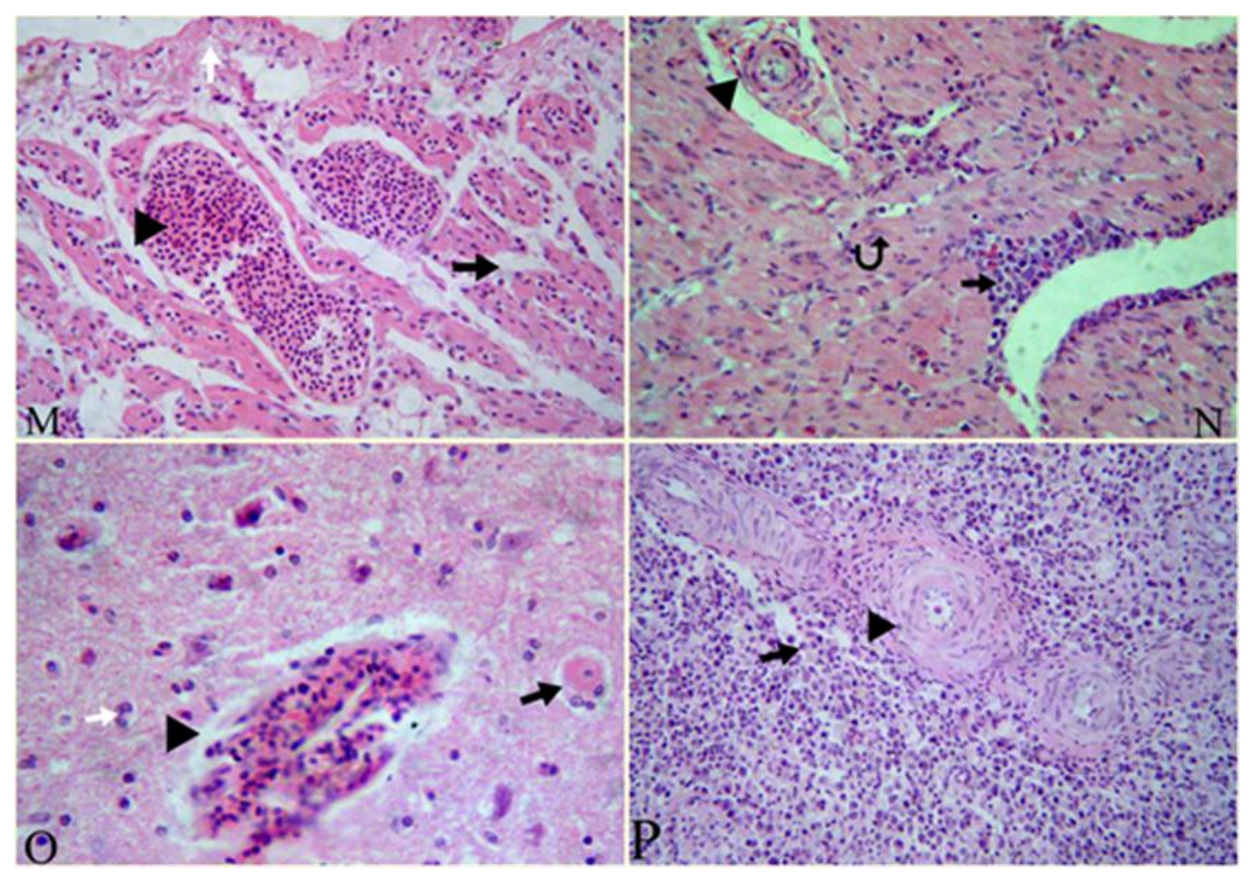

Fig. 4: (M) The heart showing thickening in the pericardium with lymphocytes infiltration and fibrin thread (white arrow). Congestion of blood vessels (arrow head) with intermuscular edema between muscle fibers (arrow) HE x1200. (N) the heart showing focal aggregations of lymphocytes and heterophils infiltrations with hemorrhage (arrow). Thickening in the wall of blood vessels with perivascular edema (arrow head) HE x1200. (O) The brain showing perivascular lymphocytic cuffing (arrow head) with satellitosis (black arrow) and neurophagia (whit arrow) HE x1200. (P) The spleen showing hyaline thickening of central arterioles with endotheliosis (arrow head) and lymphocytes depletion (arrow) HE x1200.

\section{DISCUSSION}

Infectious bronchitis virus (IBV) is prevalent in all countries with intensive poultry industry. Infectious bronchitis is a highly contagious acute viral disease infecting all ages of broiler and layer chickens causing respiratory and urogenital manifestations [17]. The outbreaks of IB are due to continuous emergence of variants of IBV strain. In the present study, the most observed 
clinical signs were depression, reduced feed consumption, ruffled feather, huddle together under heat source, coughing, gasping, sneezing and tracheal rales. Similar results were reported in a previous study [18] which stated that the first replication of the virus was in the respiratory tissues causing the above mentioned characteristic respiratory signs. Histopathological examination of diseased broilers` tissues was a helpful tool for diagnosis of the disease. Macroscopically, the trachea showed congestion and catarrhal exudate in its lumen. The airsac was cloudy with fibrin thread. Pneumonic area has been observed in the lungs. Kidneys were enlarged, inflamed and ureters were distended with urates which were similar to a previously published data [6, 11]. Microscopically, the trachea (the predilection site of the virus) showed catarrhal tracheitis and necrosis of epithelial lining as declared previously [19]. The kidneys showed congestion of peritubular blood vessels with hemorrhage. Dilation of Bowman's capsule with necrosis of some renal tubules characterized by pyknosis, karyorhexsis and karyolysis were detected. Interstitial lymphocytic and fibroblast infiltrations were observed. Cellular and hyaline cast inside the lumen of some renal tubules were detected. Similar microscopic examination of Kidneys was also reported in another study [12]. Hemorrhage and congestion with heterophils` and lymphocytes` infiltrations were detected in the air sac, liver, lungs, heart, muscle, ovary and brain. Our findings were partially agreed with those published by Gola and co-authors [12] who described the lesion of air sac edema, epithelial desquamation and fibrinous exudate. Infectious bronchitis virus spread in the Middle East between the countries through crossborder movements of poultry and poultry-related products [20]. The differential diagnosis of IB from other espiratory diseases, such as Newcastle disease (ND), infectious coryza, avian influenza (AI) and infectious laryngotracheitis was reported. It was found that head swelling was characteristic in coryza, high mortality in AI and neurological signs and diarrhoea in ND, and all these symptoms are uncommon in IBV infection [21]. Herein, RT-PCR confirmed the infectious bronchitis infection by detecting of IBV in examined organs of infected broilers. Our finding was consistent with a previous study [22] in which the classic methods for IBV diagnosis including serological or virus isolation were slow and time consuming, on the contrary, RT- PCR gave accurate, rapid and sensitive diagnostic results when used in detection of IBV.

The control of IBV occurred by live and inactivated vaccine strains that are similar field strain virus. Vaccination programmes consisting of variable strains was used to induce a broader and higher protection against challenges with several heterologous strains of IB [23].

\section{CONCLUSION}

Infectious bronchitis is an acute, highly contagious viral disease of respiratory and urogenital system affecting both broilers and layers. It caused a great economic loss to poultry industry worldwide. The histopathology and PCR technique were the most accurate method for diagnosis of IBV.

\section{ACKNOWLEDGEMENT}

We are grateful to Dr. Ahmed Erfan, Head of Biotechnology Unit, Reference Laboratory for Veterinary Quality Control on Poultry Production, Animal Health Research Institute, Dokki, Giza, Egypt for his valuable assistance in performing PCR technique.Funding Information:

\section{CONFLICT OF INTEREST}

Authors declare no conflict of interest

\section{REFERENCES}

1. Cavanagh, D. and J. Gelb, 2008. Diseases of poultry. 117-135.

2. Lai, M. M. and D. Cavanagh, 1997.The molecular biology of coronaviruses. Advances in virus research. 48: 1-100.

3. Cook, J. K., M. Jackwood and R. C. Jones,2012. The long view: 40 years of infectious bronchitis research. Avian pathology: journal of the W.V.P.A. 41(3): 239-250.

4. Schalk, A. F., 1931.An apparently new respiratory disease of baby chicks. J Am Vet Med Assoc. 78: 413-422.

5. Jackwood, M. W., 2012.Review of infectious bronchitis virus around the world. Avian diseases. 56(4): 634-641.

6. Ziegler, A. F., B. S. Ladman, P. A. Dunn, A. Schneider, S. Davison, P. G. Miller, H. Lu, D. Weinstock, M. Salem, R. J. Eckroade and J. Gelb, Jr., 2002.Nephropathogenic infectious bronchitis in Pennsylvania chickens 1997-2000. Avian diseases. 46(4): 847-858.

7. Okino, C. H., I. L. dos Santos, F. S. Fernando, A. C. Alessi, X. Wang and H. J. Montassier, 2014.Inflammatory and cellmediated immune responses in the respiratory tract of chickens to infection with avian infectious bronchitis virus. Viral immunology. 27(8): 383-391.

8. Fernando, F. S., C. H. Okino, K. R. Silva, C. C. Fernandes, M. C. M. Gonçalves, M. F. S. Montassier, R. O. Vasconcelos and H. J. Montassier, 2015.Increased expression of Interleukin-6 related to nephritis in chickens challenged with an Avian infectious bronchitis virus variant. Pesquisa Veterinária Brasileira. 35: 216-222.

9. Bande, F., S. S. Arshad, A. R. Omar, M. H. Bejo, M. S. Abubakar and Y. Abba, 2016.Pathogenesis and Diagnostic Approaches of Avian Infectious Bronchitis. Advances in virology. 2016: 4621659.

10. Ganapathy, K., 2009. Diagnosis of infectious bronchitis in chickens. In Practice. 31(9): 424-431.

11. Feng, J., Y. Hu, Z. Ma, Q. Yu, J. Zhao, X. Liu and G. Zhang, 2012.Virulent Avian Infectious Bronchitis Virus, People's Republic of China. Emerging Infectious Diseases. 18(12): 1994-2001. 
12. Gola, S., S.K.Shukla, S. Shekhar and M. Kumar, 2017. Prevalence, Sero- diagnosis and Histopathological Changes in Field Cases of Infectious Bronchitis in Chickens. Int J Curr Microbiol App Sci. 6(8): 1591-1597.

13. BochkovY.A., G.V. Batchenko, L.O. Shcherbakova, A.V. Borisov, V. V. Drygin, 2006. Molecular epizootiology of avian infectious bronchitis in Russia. Avian Pathol. 35:379-393

14. Huo, Y.F., Q.H. Huan., M. Lu, J.Q. Wu, S.Q. Lin, F.Z. Zhu, X.M. Zhang, Y.Y. Huang, S.H. Yang, C.T. Xu, 2016. Attenuation mechanism of virulent infectious bronchitis virus strain with QX genotype by continuous passage in chicken embryos. j.Vaccine. 34:83-89.

15. Bancroft, J., A. Floyd and S. Suvarna, 2013. Bancroft's theory and practice of Histological techniques. 7th ed., Churchill Livingstone. Elsevier, England 2013.

16. Meir, R., O. Maharat, Y. Farnushi and L. Simanov, 2010. Development of a real-time TaqMan RT-PCR assay for the detection of infectious bronchitis virus in chickens, and comparison of RT-PCR and virus isolation. Journal of virological methods. 163(2): 190-194.

17. Cumming, R. B. Infectious avian nephrosis (uremia) in Australia, 2008.

18. Capua, I., R. Gough, M. Mancini, C. Casaccia and C. Weiss, 1994. A novel infectious bronchitis strain infecting broiler chickens in Italy. J Vet Med B., . 41(1-10): 83-89.

19. Chousalkar, K. K., J. R. Roberts and R. Reece, 2007. Histopathology of two serotypes of infectious bronchitis virus in laying hens vaccinated in the rearing phase. Poultry science. 86(1): 59-62.

20. Abdel-Moneim, A. S., M. F. El-Kady, B. S. Ladman and J. Gelb, 2006. S1 gene sequence analysis of a nephropathogenic strain of avian infectious bronchitis virus in Egypt. Virology Journal. 3: 78-78.

21. Droual, R. and P. Woolcock, 1994. Swollen head syndrome associated with E. coli and infectious bronchitis virus in the Central Valley of California. Avian Pathology. 23(4): 733-742.

22. Kwon, H. M., M. W. Jackwood and J. Gelb, Jr., 1993. Differentiation of infectious bronchitis virus serotypes using polymerase chain reaction and restriction fragment length polymorphism analysis. Avian diseases. 37(1): $194-202$.

23. Cook J. K., S. J. Orbell, M.A. Woods, M. B. Huggins, 1999. Breadth of protection of the respiratory tract provided by different live-attenuated infectious bronchitis vaccines against challenge with infectious bronchitis viruses of heterologous serotypes. Avian. Pathology. 28: 477-485. 